\title{
ENDOMORPHISM RINGS OF BUTLER GROUPS
}

\author{
D. M. ARNOLD and C. I. VINSONHALER
}

(Received 20 June 1985)

Communicated by $\mathbf{H}$. Lausch

\begin{abstract}
This note is devoted to the question of deciding whether or not a subring of a finite-dimensional algebra over the rationals, with additive group a Butler group, is the endomorphism ring of a Butler group (a Butler group is a pure subgroup of a finite direct sum of rank-1 torsion-free abelian groups). A complete answer is given for subrings of division algebras. Several applications are included.
\end{abstract}

1980 Mathematics subject classification (Amer. Math. Soc.): $20 \mathrm{~K} 15$.

A. L. S. Corner, in 1963, proved that each reduced subring of a finite-dimensional $Q$-algebra is isomorphic to the endomorphism ring of a finite-rank torsion-free abelian group, where $Q$ denotes the field of rationals. M. C. R. Butler, in 1965, defined a class of finite-rank torsion-free abelian groups, subsequently called Butler groups. This class is the smallest class that contains all rank-1 torsion-free abelian groups and is closed under pure subgroups, torsion-free homomorphic images, and finite direct sums ([6]).

In 1965, S. Brenner and M. C. R. Butler showed that each finite-dimensional $Q$-algebra is isomorphic to the quasi-endomorphism ring of a Butler group, where the quasi-endomorphism ring of $G$ is the tensor product of $Q$, over $Z$, with the endomorphism ring of $G$. Furthermore, the additive group of the endomorphism ring of a Butler group is again a Butler group ([6]). A partial converse to the latter result is:

Theorem I. Assume that $R$ is a subring of $K=Q R$, a finite-dimensional $Q$-algebra and that the additive group of $R$ is a Butler group.

(a) If $R$ is p-reduced for at least 5 primes of $Z$, then there is a Butler group $G$ with endomorphism ring isomorphic to $R$.

(C) 1987 Australian Mathematical Society $0263-6115 / 87 \$ A 2.00+0.00$ 
(b) The group $G$ may be chosen with rank $=2 m \operatorname{rank}(R)$, where $m-1$ is the cardinality of a set of $Q$-algebra generators of $K$ containing $1_{K}$.

A Butler group $G$ is a $B_{0}$-group (called torsionless in [6]) if $G^{*}(\tau)$ is a pure subgroup of $G$ for each type $\tau$, where $G^{*}(\tau)$ is the subgroup of $G$ generated by $\left\{x \in G \mid\right.$ type $\left._{G}(x)>\tau\right\}$. Among the class of almost completely decomposable groups, the $B_{0}$-groups are precisely the completely decomposable groups. Thus, the endomorphism ring of an indecomposable, almost completely decomposable Butler group of rank $>1$ cannot be isomorphic to the endomorphism ring of a $B_{0}$-group in this restricted class. On the other hand, if the endomorphism ring of a Butler group is a subring of a division algebra, then the following theorem shows that the ring is also the endomorphism ring of a $B_{0}$-group.

THEOREM II. Assume that $R$ is a subring of $K=Q R$, a finite-dimensional division $Q$-algebra. The following statements are equivalent:

(a) $R$ is isomorphic to the endomorphism ring of a Butler group;

(b) $R$ is isomorphic to the endomorphism ring of a $B_{0}$-group;

(c) $R$ is a free $S$-module for some rank-1 torsion-free ring $S$; if $R$ is p-reduced for at most 4 primes of $Z$, then $K=Q(\gamma)$ for some $\gamma$; and if $R$ is p-reduced for at most 3 primes of $Z$, then $K=Q$.

As a consequence of Theorem II, not every subring of a finite-dimensional $Q$-algebra, with additive group a Butler group, is isomorphic to the endomorphism ring of a Butler group. For example, $R=H\left(Z_{p}\right)$, the ring of Hamiltonian quaternions over the integers localized at a prime $p$ fails to satisfy Theorem II (c). Applications of Theorem II are given in Examples 2 and 3.

If $G$ is a Butler group, then typeset $(G)$ is finite and $\left\langle G^{*}(\tau)\right\rangle_{*} / G^{*}(\tau)$ is finite for each type $\tau$, where $\left\langle G^{*}(\tau)\right\rangle_{*}$ is the pure subgroup of $G$ generated by $G^{*}(\tau)$ ([6]). It is conjectured in [6], that each Butler group contains a $B_{0}$-group as a subgroup of finite index. This conjecture is resolved, in the negative, by Example 4.

Notation and terminology are, unless otherwise noted, as in [1] and [2]. We write $M^{n}$ for the direct sum of $n$ copies of $M$ and $\operatorname{End}_{R}(M)$ for the ring of $R$-endomorphisms of an $R$-module $M$. If $S \subseteq M$, then $R S$ denotes the $R$-submodule of $M$ generated by $S$.

A torsion-free abelian group is completely decomposable (of finite rank) if it is isomorphic to a finite direct sum of subgroups of $Q$ and almost completely decomposable if it contains a completely decomposable group as a subgroup of finite index. 
Let $G$ be a torsion-free abelian group of finite rank. Then $G$ is $p$-reduced for a prime $p$ of $Z$ if $G$ contains no elements of infinite $p$-height. If $\tau$ is a type, then $G(\tau)=\left\{x \in G \mid \operatorname{type}_{G}(x) \geqslant \tau\right\}$ is a pure fully invariant subgroup of $G$. Thus, $G^{*}(\tau)$ is the subgroup of $G$ generated by $\{G(\sigma) \mid \sigma>\tau\}$. Define typeset $(G)=$ type $_{G}(x) \mid 0 \neq x \in G$ \}. The group $G$ is homogeneous if typeset $(G)$ has cardinality 1 .

LEMMA 1 (BRENNER [4]). Let $K$ be a finite-dimensional $Q$-algebra and let $m-1$ be the cardinality of a set of $Q$-algebra generators for $K$ that contains $1_{K}$.

(a) There are five left $K$-submodules of $K^{2 m}$ such that $K$ is isomorphic to the algebra of $Q$-endomorphisms of $K^{2 m}$ that leave each of the submodules invariant.

(b) If $m-1=2$, then there are four left $K$-submodules of $K^{2}$ such that $K$ is isomorphic to the algebra of $Q$-endomorphisms of $K^{2}$ that leave each of the submodules invariant.

Proof. An explicit construction of these submodules is given for later reference.

(a) Define $m \times 2 m$ matrices as follows: $M_{1}=(I O), M_{2}=(O I), M_{3}=(I I)$, $M_{4}=(I J)$, and $M_{5}=(I M)$, where $I$ is an $m \times m$ identity matrix, $O$ is an $m \times m$ zero matrix, $J$ is an $m \times m$ Jordan matrix with ones on the superdiagonal and zeros elsewhere, $M$ is an $m \times m$ matrix of the form

$$
M=\left(\begin{array}{lllllll}
0 & 0 & 0 & \cdots & 0 & 0 & 0 \\
1 & 0 & 0 & \cdots & 0 & 0 & 0 \\
\gamma_{2} & 1 & 0 & \cdots & 0 & 0 & 0 \\
0 & \gamma_{3} & 1 & \cdots & 0 & 0 & 0 \\
\vdots & \vdots & \vdots & & \vdots & \vdots & \vdots \\
0 & 0 & 0 & \cdots & 1 & 0 & 0 \\
0 & 0 & 0 & \cdots & \gamma_{m-1} & 1 & 0
\end{array}\right)
$$

and $\Gamma=\left\{\gamma_{1}=1, \gamma_{2}, \ldots, \gamma_{m-1}\right\}$ is a set of $Q$-algebra generators of $K$. Define $K_{i}$ to be the $K$-submodule of $K^{2 m}$ generated by the rows of $M_{i}$.

Note that left multiplication induces a well-defined algebra injection from $K$ to

$$
\left\{f \in \operatorname{End}_{Q}\left(K^{2 m}\right) \mid f\left(K_{i}\right) \subseteq K_{i}, 1 \leqslant i \leqslant 5\right\} .
$$

Next, let $f \in \operatorname{End}_{Q}\left(K^{2 m}\right)$ with $f\left(K_{i}\right) \subseteq K_{i}$ for each $i$. Represent $f$ as a $2 m \times 2 m$ matrix with entries in $\operatorname{End}_{Q}(K)$, say,

$$
f=\left(\begin{array}{ll}
N_{1} & N_{2} \\
N_{3} & N_{4}
\end{array}\right)
$$


$N_{1}=N_{4}$, since $f\left(K_{3}\right) \subseteq K_{3} ; N_{1}$ is a lower triangular matrix with equal diagonal elements and equal subdiagonal elements, since $f\left(K_{4}\right) \subseteq K_{4} ; N_{1}$ is a diagonal matrix with equal diagonal elements, since $f$ sends the first row of $M_{5}$ into $K_{5}$; and there is $k \in K$ with $f$ acting as left multiplication by $k$, since $f\left(K_{5}\right) \subseteq K_{5}$ and $\Gamma$ is a set of $Q$-algebra generators for $K$. For the latter statement, observe that if $f=\alpha I_{2 m \times 2 m}$ for some $\alpha \in \operatorname{End}_{Q}(K)$, then $\alpha\left(k \gamma_{i}\right)=\alpha(k) \gamma_{i}$ for each $k \in K, 2 \leqslant i \leqslant m-1$, since $f\left(K_{5}\right) \subseteq K_{5}$. Therefore, $f$ is left multiplication by $\alpha(1)$, as desired.

(b) Let $\Gamma=\{1, \gamma\}, x_{1}=(1,0), x_{2}=(0,1), x_{3}=(1,1)$, and $x_{4}=(1, \gamma)$. Define $K_{i}$ to be the $K$ - submodule of $K^{2}$ generated by $x_{i}$. Then, as in the proof of (a), left multiplication induces an isomorphism $K \rightarrow\left\{f \in \operatorname{End}_{Q}\left(K^{2}\right) \mid f\left(K_{i}\right) \subseteq K_{i}\right.$, $1 \leqslant i \leqslant 4\}$.

Proof of Theorem I. Choose distinct primes $p_{1}, p_{2}, p_{3}, p_{4}, p_{5}$ such that $R$ is $p_{i}$-reduced. Let $X_{j}$ be the subgroup of $Q 1_{K} \subseteq K$ generated by $\left\{1_{K} / p_{i}^{k} \mid 1 \leqslant i \neq j\right.$ $\leqslant 5, k=1,2, \ldots\}$. Define $B=\left(X_{1} R\right)^{m} \oplus \cdots \oplus\left(X_{5} R\right)^{m} \subseteq K^{5 m}$. Then $B$ is a Butler group, since $R$ is a Butler group, noting that $X_{1} R \simeq X_{1} \otimes_{z} R$ and $X_{1}$ is a flat $Z$-module.

Next, define $G=$ Image $\Phi \cap B$, where $\Phi: K^{2 m} \rightarrow K^{5 m}$ is given by $\Phi(x, y)=$ $(y, x, y-x, y-x \alpha, y-x \gamma), \quad y \in K^{m}, \quad x=\left(x_{1}, \ldots, x_{m}\right) \in K^{m}, \quad x \alpha=$ $\left(0, x_{1}, x_{2}, \ldots, x_{m-1}\right), \quad x \gamma=\left(x_{2}+x_{3} \gamma_{2}, x_{3}+x_{4} \gamma_{3}, \ldots, x_{m}, 0\right)$, and $\Gamma=\{1=$ $\left.\gamma_{1}, \gamma_{2}, \ldots, \gamma_{m-1}\right\} \subseteq R$ is a set of $Q$-algebra generators for $K=Q R$. Then $G$ is a Butler group, being a pure subgroup of $B$. Moreover, $\operatorname{rank}(G)=\operatorname{dim}_{Q}(\operatorname{Image} \Phi)$ $=2 m \operatorname{dim}_{Q}(K)=2 m \operatorname{rank}(R)$, since $Q B=K^{5 m}, \Phi$ is an injection, and $Q R=K$.

It is sufficient to prove that left multiplication induces an isomorphism $\mu$ : $R \rightarrow \operatorname{End}(G)$. Note that $R B=B$ and $\mu$ is a well-defined ring injection. The strategy is to first prove that if $f \in \operatorname{End}(G)$, then $f$ is left multiplication by some $k \in K$ and then to prove that $k \in R$.

Define $\tau_{j}=\operatorname{type}\left(X_{j}^{*} S\right)$ for each $1 \leqslant j \leqslant 5$, where $S$ is the pure subgroup of $R$ generated by $1_{R}=1_{K}$, and $X_{j}^{*}=\bigcap\left\{X_{i} \mid 1 \leqslant i \neq j \leqslant 5\right\}=Z\left[1 / p_{j}\right] \cdot 1_{K}$. Since $G$ is a pure subgroup of $B$, then $G\left(\tau_{j}\right)=G \cap B\left(\tau_{j}\right)$, is a fully invariant subgroup of $G$. In fact, $B\left(\tau_{j}\right)=\oplus\left\{\left(X_{i} R\right)^{m} \mid 1 \leqslant i \neq j \leqslant 5\right\}$, since if $i \neq j$ then $\tau_{j} \leqslant \operatorname{type}(x)$ for each $x \in X_{i} R$ while $\tau_{j} \nless \operatorname{type}(x)$ for each non-zero $x$ in $X_{j} R$. Consequently, $Q G\left(\tau_{j}\right)=\Phi\left(K_{j}\right)$, where $K_{j}$ is defined as in Lemma 1.a, since $\Phi\left(K_{j}\right)=$ $\left\{\Phi(x, y) \mid(x, y) \in K^{2 m}\right.$ and the $j$ th coordinate of $\left.\Phi(x, y)=0\right\}$.

Now let $f \in \operatorname{End}(G)$. Then $f\left(G\left(\tau_{j}\right)\right) \subseteq G\left(\tau_{j}\right)$, whence $f\left(Q G\left(\tau_{j}\right)\right) \subseteq Q G\left(\tau_{j}\right)$, for each $1 \leqslant j \leqslant 5$. Therefore, by Lemma 1 and the fact that $\Phi\left(K_{j}\right)=Q G\left(\tau_{j}\right), f$ is left multiplication by some $k \in K$.

To prove that $k \in R$, let $x=y=(1,0, \ldots, 0) \in K^{m}$. Then $\Phi(0, y) \in G\left(\tau_{2}\right)$, $f(\Phi(0, y))=k \Phi(0, y)=(k y, 0, k y, k y, k y) \in G\left(\tau_{2}\right) \subseteq B$, and $k \in X_{1} R \cap X_{3} R$ $\cap X_{4} R \cap X_{5} R$. Similarly, $k \Phi(x, 0) \in G\left(\tau_{1}\right)$ implies that $k \in X_{2} R$. Thus, $k \in$ 
$\bigcap\left\{X_{i} R \mid 1 \leqslant i \leqslant 5\right\}=R$, since $Z \cdot 1_{K}=\bigcap\left\{X_{i} \mid 1 \leqslant i \leqslant 5\right\}$. Consequently, $k \in R$, as desired.

Proof of TheOREM II. (b) $\Rightarrow$ (a) is clear.

(a) $\Rightarrow$ (c). Assume that $R=\operatorname{End}(G)$ for some Butler group $G$. Then $R$ is a free $S$-module, where $S$ is the pure subgroup of $R$ generated by $1_{R}$, by [2], Corollary 5.2. Note that if $p$ is a prime, then $R$ is $p$-reduced if and only if $R$ is not $p$-divisible, whence $R$ is $p$-reduced if and only if $G$ is not $p$-divisible.

It is now sufficient to prove that if $R$ is divisible by all but four, respectively three, primes, then there are four, respectively three, $Q R$-submodules of $Q G$ such that $Q R$ is the algebra of $Q$-endomorphisms of $Q G$ that leave each of these submodules invariant. In this case, $Q R=Q(\gamma)$, respectively $Q R=Q$, as a consequence of [4], Proposition 5 and Section 7.

The case that $G$ is divisible by all but four primes, say $p_{1}, p_{2}, p_{3}, p_{4}$, is proved and the other case, having an analogous proof, is left to the reader.

The four desired submodules of $Q G$ are $L_{i}=Q G\left(\tau_{i}\right)$ for $1 \leqslant i \leqslant 4$, where $\tau_{j}=\inf \left\{\sigma_{i} \mid 1 \leqslant i \neq j \leqslant 4\right\}, \sigma_{i}=\operatorname{type}\left(Z_{p_{i}}\right)$, and $Z_{p_{i}}$ is the localization of $Z$ at $p_{i}$. To see this is indeed the case, first note that typeset $(G) \subseteq T$, the finite sublattice of the lattice of all types generated by $\sigma_{1}, \sigma_{2}, \sigma_{3}, \sigma_{4}$. Then $\tau_{1}, \tau_{2}, \tau_{3}, \tau_{4}$ are minimal elements of $T \backslash\left\{\tau_{0}\right\}$, where $\tau_{0}=\inf \left\{\sigma_{1}, \sigma_{2}, \sigma_{3}, \sigma_{4}\right\}$, and $\sigma_{1}, \sigma_{2}, \sigma_{3}, \sigma_{4}$ are maximal elements of $T \backslash\{$ type $(Q)\}$.

Since typeset $(G) \subseteq T, G$ is a pure subgroup of $C=C_{1} \oplus \cdots \oplus C_{n}$, with $C_{i} \subseteq Q$ and type $\left(C_{i}\right) \in T([6])$. For each $1 \leqslant i \leqslant 4, \operatorname{typeset}\left(C\left(\sigma_{i}\right)\right) \subseteq\left\{\sigma_{i}, \operatorname{type}(Q)\right\}$ so that $G\left(\sigma_{i}\right)$ is a summand of $C\left(\sigma_{i}\right)$ ([1], Exercise 5.7), hence of $G$. Therefore, $G\left(\sigma_{i}\right)=0$, since $Q \operatorname{End}(G) \simeq K$, a division algebra, implies that $G$ is indecomposable. It now follows that typeset $(G) \subseteq\left\{\tau_{0}, \tau_{1}, \tau_{2}, \tau_{3}, \tau_{4}, \tau^{i j} \mid 1 \leqslant i \neq j \leqslant 4\right\}$, where $\tau^{i j}=\sup \left\{\tau_{i}, \tau_{j}\right\}=\inf \left\{\sigma_{k}, \sigma_{l}\right\}$, and $\{i, j, k, l\}=\{1,2,3,4\}$.

Finally, assume that $f \in$ End $_{Q}(Q G)$, with $f\left(L_{i}\right) \subseteq L_{i}$ for each $1 \leqslant i \leqslant 4$. Then $f\left(Q G\left(\tau^{i j}\right)\right) \subseteq Q G\left(\tau^{i j}\right)$, for each $1 \leqslant i \neq j \leqslant 4$, noting that $Q G\left(\tau^{i j}\right)=Q G\left(\tau_{i}\right) \cap$ $Q G\left(\tau_{j}\right)$ for each $\tau \in \operatorname{typeset}(G)$. Therefore, $f \in Q \operatorname{End}(G)$ by [2], Theorem 1.5. Consequently, left multiplication induces an isomorphism $Q R \rightarrow\{f \in$ End $\left._{Q}(Q G) \mid f\left(L_{i}\right) \subseteq L_{i}, 1 \leqslant i \leqslant 4\right\}$, as desired.

(c) $\Rightarrow$ (b). If $R$ is $p$-reduced for at most 3 primes, then $K=Q$, by hypothesis. In this case, the additive group of $R$ is a $B_{0}$-group with endomorphism ring $R$.

The next case considered is that $R$ is $p$-reduced for at least 5 primes. The construction of Theorem I yields a Butler group $G=\operatorname{Image} \Phi \cap B$ with $R=$ $\operatorname{End}(G)$, where $B=\left(X_{1} R\right)^{m} \oplus \cdots \oplus\left(X_{5} R\right)^{m} \subseteq K^{5 m}$ and $\Gamma=\{1=$ $\left.\gamma_{1}, \ldots, \gamma_{m-1}\right\}$ is a set of $Q$-algebra generators of $K$, which may be asssumed to be a subset of $R$ since $K=Q R$. We prove that $G$ is a $B_{0}$-group. 
Since $R$ is a free $S$-module, $X_{i} R$ is homogeneous completely decomposable with type $=\sigma_{i}=\operatorname{type}\left(X_{i} S\right)$. Recall, from the proof of Theorem I, that $X_{j}^{*}=$ $\bigcap\left\{X_{i} \mid 1 \leqslant i \neq j \leqslant 5\right\}, \tau_{j}=\operatorname{type}\left(X_{j}^{*} S\right)$, and $\Phi\left(K_{j}\right)=Q G\left(\tau_{j}\right)$, where $K_{j}$ is as defined in Lemma 1.a.

A routine calculation shows that if $1 \leqslant i \neq j \leqslant 5$, then $K_{i} \cap K_{j}=0$, unless $\{i, j\}=\{1,4\}$ or $\{1,5\}$. Consequently, typeset $(G) \subseteq\left\{\tau_{0}, \tau_{1}, \tau_{2}, \tau_{3}, \tau_{4}, \tau_{5}, \tau^{14}, \tau^{15}\right\}$, where type $(S)=\tau_{0}=\inf \left\{\tau_{1}, \tau_{2}, \tau_{3}, \tau_{4}, \tau_{5}\right\}$ and $\tau^{i j}=\sup \left\{\tau^{i}, \tau^{j}\right\}$.

It is now sufficient to prove that $G^{*}\left(\tau_{0}\right\}=G$ and that $G^{*}\left(\tau_{1}\right)$ is pure in $G$, in which case $G$ must be a $B_{0}$-group. In fact, $G^{*}\left(\tau_{1}\right)=G\left(\tau^{14}\right)+G\left(\tau^{15}\right)$ is pure in $G$, since $K_{1} \cap K_{4}=\Phi^{-1} Q G\left(\tau^{14}\right)=Q(0,0, \ldots, 0,1,0,0, \ldots, 0)$ and $K_{1} \cap K_{5}=$ $\Phi^{-1} Q G\left(\tau^{15}\right)=Q(1,0, \ldots, 0,0,0, \ldots, 0)$.

Note that $G^{*}\left(\tau_{0}\right)=G\left(\tau_{1}\right)+G\left(\tau_{2}\right)+G\left(\tau_{3}\right)+G\left(\tau_{4}\right)+G\left(\tau_{5}\right)$. To see that $G^{*}\left(\tau_{0}\right)=G$, it is sufficient to prove that if $p$ is a prime, then $Z_{p} \otimes_{Z} G=G_{p} \subseteq$ $G\left(\tau_{1}\right)_{p}+\cdots+G\left(\tau_{5}\right)_{p}$, in which case, $G=\bigcap_{p} G_{p} \subseteq G^{*}\left(\tau_{0}\right)=\bigcap_{p} G^{*}\left(\tau_{0}\right)_{p}$. Let $a=(y, x, y-x, y-x \alpha, y-x \gamma) \in G_{p} \subseteq\left(X_{1} R\right)_{p}^{m} \oplus \cdots \oplus\left(X_{5} R\right)_{p}^{m}$. If $p \notin$ $\left\{p_{3}, p_{4}, p_{5}\right\}$, then $y \in\left(X_{1} R\right)_{p}^{m}=\left(X_{2}^{*} R\right)_{p}^{m}$ and $x \in\left(X_{2} R\right)_{p}^{m}=\left(X_{1}^{*} R\right)_{p}^{m}$. Hence, $a=\Phi(x, y)=\Phi(0, y)+\Phi(x, 0) \in G\left(\tau_{2}\right)_{p}+G\left(\tau_{1}\right)_{p}$. Similarly, if $p=p_{3}$, then $a=\Phi(0, y-x)+\Phi(x, x) \in G\left(\tau_{2}\right)_{p}+G\left(\tau_{3}\right)_{p} ;$ if $p=p_{4}$, then $a=$ $\Phi(0, y-x \alpha)+\Phi(x, x \alpha) \in G\left(\tau_{2}\right)_{p}+G\left(\tau_{4}\right)_{p} ;$ and if $p=p_{5}$, then $a=$ $\Phi(0, y-x \gamma)+\Phi(x, x \gamma) \in G\left(\tau_{2}\right)_{p}+G\left(\tau_{5}\right)_{p}$. Consequently, $G^{*}\left(\tau_{0}\right)=G$, as desired.

The final case is that $R$ is $p$-reduced for exactly four primes. The proof, using Lemma 1.b in place of Lemma 1.a, is similar, but easier, and thus is omitted.

EXAMPLE 2. There is a $B_{0}$-group $A$ with $\operatorname{End}(A) \simeq Z \oplus 2 Z i \subseteq Q(i)$, where $i^{2}=-1$. In particular, $\operatorname{End}(A)$ is not integrally closed in its quotient field $Q(i)$.

Example 2 and Theorem II are a partial resolution of Problem 6.5, [2]. The following example is a counterexample to Conjecture 6.2, [2].

EXAmple 3. There are $B_{0}$-groups $A$ and $B$ such that $A$ and $B$ are nearly isomorphic but not isomorphic.

Proof. Let $S=Z[\sqrt{-5}]$, a Dedekind domain that is not a principal ideal domain. By Theorem II, there is a $B_{0}$-group $A$ with $\operatorname{End}(A)=S$ I at $I$ he a non-principal ideal of $S$. Then $B=I A$ is a subgroup of finite index in $A, A$ and $B$ are nearly isomorphic, but $A$ and $B$ are not isomorphic (as in [1], Example 12.11).

EXAMPLE 4. There is a rank-4 Butler group that does not contain a $B_{0}$-group as a subgroup of finite index. 
Proof. Let $V=Q v_{1} \oplus Q v_{2} \oplus Q v_{3} \oplus Q v_{4}$ and let $\left\{p_{1}, \ldots, p_{9}\right\}$ be a set of distinct primes. Denote by $Z\left[1 / p_{i}\right]$ the subring of $Q$ generated by $1 / p_{i}$, and define $Z^{i j}=Z\left[1 / p_{i}\right]+Z\left[1 / p_{j}\right]$ whenever $1<i \neq j \leqslant 9$. Let $G$ be the subgroup of $V$ generated by $\left\{A_{1}, \ldots, A_{6}\right\}$, where $A_{1}=Z^{15} v_{1}, A_{2}=Z^{25} v_{2}, A_{3}=Z^{36} v_{3}$, $A_{4}=Z^{46} v_{4}, A_{5}=Z^{79}\left(v_{1}+v_{2}+p_{9} v_{3}\right)$, and $A_{6}=Z^{89}\left(v_{3}+v_{4}+p_{9} v_{1}\right)$. Then each $A_{i}$ is a pure rank-1 subgroup of $G$ and typeset $(G)=\left\{\tau_{1}, \ldots, \tau_{6}, \tau, \tau_{12}, \tau_{34}, \tau_{56}\right\}$, where $\tau_{i}=\operatorname{type}\left(A_{i}\right), \tau=\operatorname{type}(Z)$ and $\tau_{i j}=\inf \left\{\tau_{i}, \tau_{j}\right\}$ (see [2], Theorems 0.1 and 1.7).

Note that $G\left(\tau_{i}\right)=A_{i}, G^{*}\left(\tau_{12}\right)=A_{1}+A_{2}$, and $G^{*}\left(\tau_{34}\right)=A_{3}+A_{4}$. Since $G\left(\tau_{12}\right) \cap G\left(\tau_{34}\right)=0, \quad G\left(\tau_{12}\right)=\left(Q v_{1} \oplus Q v_{2}\right) \cap G=A_{1}+A_{2}+Z\left(v_{1}+v_{2}\right) / p$ and $G\left(\tau_{34}\right)=A_{3}+A_{4}+Z\left(v_{3}+v_{4}\right) / p$, where $p=p_{9}$. Similarly, $G^{*}\left(\tau_{56}\right)=$ $G\left(\tau_{\mathrm{s6}}\right)$.

Suppose that $H$ is a subgroup of finite index in $G$ and that $H$ is a $B_{0}$-group. Then typeset $(H)=\operatorname{typeset}(G)$, so there exist non-zero integers $m_{i}$, such that $H=m_{1} A_{1}+\cdots+m_{6} A_{6}$ and $m_{i} A_{i}=H\left(\tau_{i}\right)$, since $H\left(\tau_{i}\right)=H \cap G\left(\tau_{i}\right)=H \cap A_{i}$ ([2], Theorem 2.2).

Furthermore, $m_{1} A_{1}+m_{2} A_{2}=H^{*}\left(\tau_{12}\right)=H\left(\tau_{12}\right)=H \cap G\left(\tau_{12}\right)$ and, similarly, $m_{3} A_{3}+m_{4} A_{4}=H \cap G\left(\tau_{34}\right)$. In particular, $\left(m_{3} A_{3}+m_{4} A_{4}+m_{5} A_{5}+\right.$ $\left.m_{6} A_{6}\right) \cap G\left(\tau_{12}\right) \subseteq m_{1} A_{1}+m_{2} A_{2}$ and $\left(m_{1} A_{1}+m_{2} A_{2}+m_{5} A_{5}+m_{6} A_{6}\right) \cap$ $G\left(\tau_{34}\right) \subseteq m_{3} A_{3}+m_{4} A_{4}$. Localizing the first containment at $p=p_{9}$ yields $\left(p^{e(3)} Z_{p} v_{3}+p^{e(4)} Z_{p} v_{4}+Q v_{5}+Q v_{6}\right) \cap\left(Z_{p} v_{1}+Z_{p} v_{2}+Z_{p}\left(v_{1}+v_{2}\right) / p\right) \subseteq$ $p^{e(1)} Z_{p} v_{1}+p^{e(2)} Z_{p} v_{2}$, where $e(i)=p$-height $\left(m_{i}\right)$ in $Z, v_{5}=\left(v_{1}+v_{2}+p v_{3}\right) / p$, and $v_{6}=\left(v_{3}+v_{4}+p v_{1}\right) / p$.

Note that $v_{5}-v_{3}=\left(v_{1}+v_{2}\right) / p$. Thus, $-p^{e(3)} v_{3}+p^{e(3)} v_{5}=p^{e(3)-1}\left(v_{1}+v_{2}\right)$ $\in p^{e(1)} Z_{p} v_{1}+p^{e(2)} Z_{p} v_{2}$. Hence, $e(3)-1 \geqslant e(1)$. However, by a similar argument on the localization of the second containment and the equation $v_{6}-v_{1}=$ $\left(v_{3}+v_{4}\right) / p, e(1)-1 \geqslant e(3)$. This contradiction completes the proof.

\section{References}

[1] D. Arnold, Finite rank torsion-free abelian groups and rings, Lecture Notes in Mathematics 931, (Springer-Verlag, 1982).

[2] D. Arnold, 'Pure subgroups of finite rank completely decomposable groups', Proceedings of Oberwolfach abelian group theory conference, Lecture Notes in Mathematics 874, (Springer-Verlag, 1981, pp. 1-31).

[3] D. Amold and C. Vinsonhaler, 'Pure subgroups of finite rank completely decomposable groups II', Proceedings of Hawaii abelian group theory conference, Lecture Notes in Mathematics 1006, (Springer-Verlag, 1983, pp. 97-143).

[4] S. Brenner, 'Endomorphism algebras of vector spaces with distinguished sets of subspaces', $J$. Algebra 6 (1967), 100-114.

[5] S. Brenner and M. C. R. Butler, 'Endomorphism rings of vector spaces and torsion free abelian groups', J. London Math. Soc. 40 (1965), 183-187. 
[6] M. C. R. Butler, 'A class of torsion-free abelian groups of finite rank', Proc. London Math. Soc. 15 (1965), 680-698.

[7] M. C. R. Butler, 'Torsion-free modules and diagrams of vector spaces', Proc. London Math. Soc. $18(1968), 635-652$.

[8] A. L. S. Corner, 'Every countable reduced torsion-free ring is an endomorphism ring', Proc. London Math. Soc. 13 (1963), 687-710.

[9] E. L. Lady, 'Extension of scalars for torsion free modules over Dedekind domains', Symposia Mathematica 23 (1979), 287-305.

[10] J. Koehler, 'The type set of a torsion-free group of finite rank', Illinois J. Math. 9 (1965), 66-86.

Department of Mathematical Sciences

New Mexico State University

Las Cruces, New Mexico 88003

U.S.A.
Department of Mathematics

University of Connecticut Storrs, Connecticut 06268

U.S.A. 